\title{
Editorial
}

\section{Acknowledgement to Reviewers of Hydrology in 2014}

Hydrology Editorial Office, MDPI AG, Klybeckstrasse 64, CH-4057 Basel, Switzerland

Published: 8 January 2015

The editors of Hydrology would like to express their sincere gratitude to the following reviewers for assessing manuscripts in 2014:

$\begin{array}{lll}\text { Anderson, Brian } & \text { Lee, Se-Yeun } & \text { Seager, Richard } \\ \text { Andreadis, Konstantinos } & \text { Li, Fadong } & \text { Sentelhas, Paulo Cesar } \\ \text { Aronica, Giuseppe T. } & \text { Liljedahl, Anna K. } & \text { Shook, Kevin } \\ \text { Badiger, Shrinivas } & \text { Lim, Kyung Jae } & \text { Singh, Shailesh Kumar } \\ \text { Biederman, Joel } & \text { Luce, Charles H. } & \text { Sola, F. } \\ \text { Brocca, Luca } & \text { Manfreda, Salvatore } & \text { Steenbergen, Niels Van } \\ \text { Cano, F. Javier } & \text { Mazvimavi, Dominic } & \text { Su, XiaoLi } \\ \text { Chau, K.W. } & \text { Momm, Henrique } & \text { Sutanudjaja, Edwin } \\ \text { Chu, Jamin } & \text { Olang, Luke } & \text { Teatini, Pietro } \\ \text { Crow, Wade } & \text { Perrin, Charles } & \text { Tøgersen, Frede Aakmann } \\ \text { Guo, Diansheng } & \text { Piniewski, Mikolaj } & \text { Van der Ploeg, Martine } \\ \text { Hossain, A.K.M. Azad } & \text { Raleigh, Mark } & \text { Wheater, Howard } \\ \text { Jeu, Richard de } & \text { Rössler, Ole } & \text { Winkler, Rita } \\ \text { Lakshmi, Venkat } & \text { Schmied, Hannes Müller } & \text { Yen, Haw }\end{array}$

(C) 2015 by the authors; licensee MDPI, Basel, Switzerland. This article is an open access article distributed under the terms and conditions of the Creative Commons Attribution license (http://creativecommons.org/licenses/by/4.0/). 